\title{
On pseudocyclic association schemes
}

\author{
Mikhail Muzychuk \\ Netanya Academic College, Netanya, Israel \\ Ilya Ponomarenko * \\ Steklov Institute of Mathematics at St. Petersburg, Russia
}

Received 15 October 2009, accepted 15 May 2011, published online 21 October 2011

\begin{abstract}
The notion of a pseudocyclic association scheme is generalized to the non-commutative case. It is proved that any pseudocyclic scheme the rank of which is much more than the valency is the scheme of a Frobenius group and is uniquely determined up to isomorphism by its intersection number array. An immediate corollary of this result is that any scheme of prime degree, valency $k$ and rank at least $k^{4}$ is schurian.
\end{abstract}

Keywords: Association schemes, Pseudocyclic schemes, Frobenius groups.

Math. Subj. Class.: 05E30,20B99

\section{Introduction}

A commutative association scheme is called pseudocyclic if the multiplicity of its nonprincipal irreducible character does not depend on the choice of the character [4] (for a background on theory of association schemes and coherent configurations we refer to [14] and Section 2). It can be proved that such a scheme is equivalenced, i.e. the valencies of its non-reflexive basis relations are pairwise equal. A classical example of a commutative pseudocyclic scheme is a cyclotomic scheme over a finite field; two other series of such schemes were constructed in [19]. A motivation to study pseudocyclic schemes is that any of them produces special 2-designs.

In this paper we define an association scheme (not necessary commutative) to be pseudocyclic if the ratio of the multiplicity and degree of its non-principal irreducible character does not depend on the choice of the character. Clearly, in the commutative case both definitions give the same concept. To formulate one of the main results of this paper (which, in

* The work was partially supported by RFFI grant 11-01-00760-a.

E-mail addresses: muzy@ netanya.ac.il (Mikhail Muzychuk), inp@pdmi.ras.ru (Ilya Ponomarenko) 
particular, shows that non-commutative pseudocyclic schemes do exist) we make two remarks. First, as in the commutative case we can prove (Theorem 3.2) that any pseudocyclic scheme is equivalenced; the valency of its non-reflexive basis relation is called the valency of the scheme. Secondly, under a Frobenius scheme we mean the scheme of a Frobenius group (in its standard permutation representation in which the one point stabilizer coincides with the Frobenius complement). Our first result is an immediate consequence of Theorems 3.1 and 7.4.

Theorem 1.1. Any Frobenius scheme is pseudocyclic. Conversely, there exists a function $f(k)$ such that any pseudocyclic scheme of valency $k>1$ and rank at least $f(k)$ is a Frobenius scheme.

The rough upper bound on the function $f(k)$ obtained in the proof is $O\left(k^{4}\right)$. On the other hand, the scheme of a non-Desarguesian affine plane of order $q$ is non-schurian (see Subsection 4.5), and hence can not be a Frobenius scheme. Besides, it is pseudocyclic of valency $q-1$ and rank $q+2$. Thus $f(k) \geq k+3$.

The proof of the second part of Theorem 1.1 is based on Theorem 5.4 giving together with Theorem 7.1 a sufficient condition for an equivalenced scheme to be schurian.

The celebrated Hanaki-Uno theorem states that any scheme of prime degree is pseudocyclic [18]. Therefore as an immediate consequence of Theorem 1.1 we have the following result.

Corollary 1.2. Any scheme of prime degree, valency $k$ and rank at least $f(k)$ is schurian.

One of the most important problems in association scheme theory is to determine a scheme up to (ordinary) isomorphism by means of the intersection number array. For example, the intersection number array of the scheme of a distance-regular graph is uniquely determined by the parameters of the graph. Therefore the most part of characterizations of the classical distance-regular graphs given in [4] are in fact the characterizations of their schemes in the above sense (we refer to [14] for a survey of relevant results). In this paper we prove the following result which is a direct consequence of Theorem 7.4.

Theorem 1.3. Any Frobenius scheme of valency $k$ and rank at least $f(k)$ is determined up to isomorphism by its intersection number array.

All undefined terms and results concerning permutation groups can be found in monographs [27, 29]. To make the paper as self-contained as possible we give a background on association schemes [30] and coherent configurations [14] in Section 2.

Section 3 contains the definition of a pseudocyclic scheme, several useful results on these schemes and the proof of the first part of Theorem 1.1 (Theorem 3.1). In Section 4 we give a brief exposition of known families of pseudocyclic schemes. In Sections 5 and 6 under a special assumption we explicitly find a one point fission of a pseudocyclic scheme and a one point fission of any algebraic isomorphism from it to another scheme (Theorems 5.4 and 6.2). Based on these results we prove our main theorems in Section 7. Section 8 includes some concluding remarks and special results concerning pseudocyclic schemes.

Notation. Throughout the paper $\Omega$ denotes a finite set. Set $1_{\Omega}=\{(\alpha, \alpha): \alpha \in \Omega\}$ and $1_{\alpha}=1_{\{\alpha\}}$ for all $\alpha \in \Omega$. For a relation $r \subset \Omega \times \Omega$ we set $r^{*}=\{(\beta, \alpha):(\alpha, \beta) \in r\}$ 
and $\alpha r=\{\beta \in \Omega:(\alpha, \beta) \in r\}$ for all $\alpha \in \Omega$. The adjacency matrix of $r$ is denoted by $A(r)$. For $s \subset \Omega \times \Omega$ we set $r \cdot s=\{(\alpha, \gamma):(\alpha, \beta) \in r,(\beta, \gamma) \in s$ for some $\beta \in \Omega\}$. If $S$ and $T$ are sets of relations, we set $S \cdot T=\{s \cdot t: s \in S, t \in T\}$. For a permutation group $G \leq \operatorname{Sym}(\Omega)$ we denote by $\operatorname{Orb}(G)=\operatorname{Orb}(G, \Omega)$ the set of $G$-orbits.

\section{Schemes, coherent configurations and permutation groups}

This section collects all definitions and basic facts related to coherent configurations and association schemes which are used in the paper. Our notation style is a mixture of the styles used in [30] and [14].

\subsection{Definitions}

Let $\Omega$ be a finite set and $S$ a partition of $\Omega \times \Omega$. Denote by $S^{\cup}$ the set of all unions of the elements of $S$. A pair $(\Omega, S)$ is called a coherent configuration on $\Omega$ if the following conditions are satisfied:

(S1) the diagonal $1_{\Omega} \subset \Omega \times \Omega$ belongs to $S^{\cup}$,

(S2) $S$ is closed with respect to $*$,

(S3) given $r, s, t \in S$, the number $c_{r s}^{t}=|\{\beta \in \Omega:(\alpha, \beta) \in r,(\beta, \gamma) \in s\}|$ does not depend on the choice of $(\alpha, \gamma) \in t$.

The elements of $\Omega, S, S^{\cup}$ and the numbers in (S3) are called the points, the basis relations, the relations and the intersection numbers of the coherent configuration $(\Omega, S)$, respectively. The numbers $|\Omega|$ and $|S|$ are called the degree and rank of it. The unique basis relation containing a pair $(\alpha, \beta) \in \Omega \times \Omega$ is denoted by $r(\alpha, \beta)$. The set of basis relations contained in $r \cdot s$ with $r, s \in S^{\cup}$ is denoted by $r s$.

\subsection{Homogeneity}

The set $\Omega$ is the disjoint union of fibers of $(\Omega, S)$, i.e. those $\Delta \subset \Omega$ for which $1_{\Delta} \in S$. For any basis relation $s \in S$ there exist uniquely determined fibers $\Delta, \Gamma$ such that $s \subset \Delta \times \Gamma$. Moreover, the number $|\delta s|$ does not depend on $\delta \in \Delta$ and coincides with $c_{s s^{*}}^{t}$ where $t=1_{\Delta}$. We denote it by $n_{s}$ and call valency of $s$. The coherent configuration $(\Omega, S)$ is called homogeneous or an association scheme or a scheme if $\Omega$ is a fiber of it. In this case

$$
n_{s}=n_{s^{*}}, \quad s \in S \text {. }
$$

We say that $(\Omega, S)$ is an equivalenced scheme of valency $k$, when $n_{s}=k$ for all $s \in S^{\#}$ where here and below we put $S^{\#}=S \backslash\left\{1_{\Omega}\right\}$.

\subsection{Isomorphisms and schurity}

Two coherent configurations are called isomorphic if there exists a bijection between their point sets preserving the basis relations. Any such bijection is called an isomorphism of these coherent configurations. The group of all isomorphisms of a coherent configuration $(\Omega, S)$ to itself (sometimes called the group of colored automorphisms) contains a normal subgroup

$$
\operatorname{Aut}(\Omega, S)=\left\{f \in \operatorname{Sym}(\Omega): s^{f}=s, s \in S\right\}
$$


called the automorphism group of $(\Omega, S)$. Conversely, let $G \leq \operatorname{Sym}(\Omega)$ be a permutation group and $S$ the set of its 2 -orbits, i.e. the orbits of componentwise action of $G$ on $\Omega \times \Omega$. Then $(\Omega, S)$ is a coherent configuration and we call it the coherent configuration of $G$. This coherent configuration is homogeneous if and only if the group $G$ is transitive; in this case we say that $(\Omega, S)$ is the scheme of $G$. A coherent configuration on $\Omega$ is called schurian if it is the coherent configuration of 2-orbits of some permutation group on $\Omega$.

\subsection{Algebraic isomorphisms and separability}

Two coherent configurations $(\Omega, S)$ and $\left(\Omega^{\prime}, S^{\prime}\right)$ are called algebraically isomorphic if

$$
c_{r s}^{t}=c_{r^{\prime} s^{\prime}}^{t^{\prime}}, \quad r, s, t \in S,
$$

for some bijection $\varphi: S \rightarrow S^{\prime}, r \mapsto r^{\prime}$ called an algebraic isomorphism from $(\Omega, S)$ to $\left(\Omega^{\prime}, S^{\prime}\right)$. Each isomorphism $f$ from $(\Omega, S)$ to $\left(\Omega^{\prime}, S^{\prime}\right)$ induces in a natural way an algebraic isomorphism between these schemes denoted by $\varphi_{f}$. The set of all isomorphisms inducing the algebraic isomorphism $\varphi$ is denoted by $\operatorname{Iso}\left(S, S^{\prime}, \varphi\right)$. In particular,

$$
\operatorname{Iso}\left(S, S, \operatorname{id}_{S}\right)=\operatorname{Aut}(\Omega, S)
$$

where $\operatorname{id}_{S}$ is the identical mapping on $S$. A coherent configurations $(\Omega, S)$ is called separable if the set $\operatorname{Iso}\left(S, S^{\prime}, \varphi\right)$ is non-empty for each algebraic isomorphism $\varphi$.

\subsection{Semiregularity}

A coherent configuration $(\Omega, S)$ is called semiregular if

$$
|\alpha s| \leq 1, \quad \alpha \in \Omega, s \in S .
$$

A semiregular scheme is called regular; regular schemes are exactly thin schemes in the sense of [30]. One can see that a coherent configuration (resp. scheme) is semiregular (resp. regular) if and only if it is a coherent configuration (resp. scheme) of a semiregular (resp. regular) permutation group. The proof of this statement as well as the next one can be found in [12].

Theorem 2.1. Any semiregular configuration is schurian and separable.

\subsection{One point fission}

Let $(\Omega, S)$ be a coherent configuration and $\alpha \in \Omega$. Denote by $S_{\alpha}$ the set of basis relations of the smallest coherent configuration on $\Omega$ such that $1_{\alpha} \in S_{\alpha}$ and $S \subset S_{\alpha}^{\cup}$ (see [14]). The coherent configuration $\left(\Omega, S_{\alpha}\right)$ is called the $\alpha$-fission (or a one point fission) of $(\Omega, S)$. It is easily seen that

$$
\operatorname{Aut}(\Omega, S)_{\alpha}=\operatorname{Aut}\left(\Omega, S_{\alpha}\right) \text {. }
$$

Notice that the set $\alpha s$ is a union of some fibers of $\left(\Omega, S_{\alpha}\right)$ for all $s \in S$, and the relation $t \cap(\alpha r \times \alpha s)$ belongs to the set $S_{\alpha}^{\cup}$ for all $r, s, t \in S$.

Let $\left(\Omega^{\prime}, S^{\prime}\right)$ be a coherent configuration and $\varphi:(\Omega, S) \rightarrow\left(\Omega^{\prime}, S^{\prime}\right)$ an algebraic isomorphism. Let $\alpha^{\prime} \in \Omega^{\prime}$ and $\psi:\left(\Omega, S_{\alpha}\right) \rightarrow\left(\Omega, S_{\alpha^{\prime}}^{\prime}\right)$ be an algebraic isomorphism such that

$$
\psi\left(1_{\alpha}\right)=1_{\alpha^{\prime}}, \quad \psi(s) \subset \varphi(\widetilde{s}), s \in S_{\alpha},
$$

where $\widetilde{s}$ is the unique basis relation of $(\Omega, S)$ that contains $s$. Then $\psi$ is uniquely determined by $\varphi$. We say that $\varphi_{\alpha, \alpha^{\prime}}:=\psi$ is the $\left(\alpha, \alpha^{\prime}\right)$-extension (or one point extension) of $\varphi$. 


\section{$2.7 t$-condition}

The following definition goes back to [15, p.70]. Let $(\Omega, S)$ be a coherent configuration. Two sets $\Delta, \Delta^{\prime} \subset \Omega$ have the same type with respect to the pair $(\alpha, \beta) \in \Omega \times \Omega$ if $\alpha, \beta \in \Delta \cap \Delta^{\prime}$ and there exists a bijection $\delta \mapsto \delta^{\prime}$ from $\Delta$ onto $\Delta^{\prime}$ such that $\alpha^{\prime}=\alpha$, $\beta^{\prime}=\beta$ and

$$
r\left(\delta_{1}, \delta_{2}\right)=r\left(\delta_{1}^{\prime}, \delta_{2}^{\prime}\right), \quad \delta_{1}, \delta_{2} \in \Delta .
$$

Let $t \geq 2$ be an integer. The coherent configuration $(\Omega, S)$ satisfies the $t$-condition at a relation $s \in S$ if for each $k=2, \ldots, t$ the number of $k$-subsets of $\Omega$ of each fixed type with respect to the pair $(\alpha, \beta) \in s$ does not depend on the choice of this pair. If the $t$ condition is satisfied at each $s \in S$, we say that $(\Omega, S)$ satisfies the $t$-condition. It can be proved that the scheme on $n$ points is schurian if and only if it satisfies the $t$-condition for all $t=2, \ldots, n-1$.

\subsection{Indistinguishing number}

Let $(\Omega, S)$ be a scheme. The indistinguishing number of a relation $s \in S$ is defined to be the number

$$
c(s)=\sum_{t \in S} c_{t t^{*}}^{s} .
$$

The term goes back to [1, p.563] where the number $n-c(s)$ with $n=|\Omega|$ was called the distinguishing number of $s$. Clearly, $c\left(1_{\Omega}\right)=n$. The maximum of $c(s), s \in S^{\#}$, is called the indistinguishing number of $(\Omega, S)$.

Lemma 2.2. Let $(\Omega, S)$ be an equivalenced scheme of valency $k$. Then the arithmetical mean of $c(s), s \in S^{\#}$, equals $k-1$.

Proof. Set $n=|\Omega|$. Then $\left|S^{\#}\right|=(n-1) / k$ and $|s|=n k$ for all $s \in S^{\#}$. Counting the number of $\Omega$-triples $(\alpha, \beta, \gamma)$ such that $r(\alpha, \beta)=r(\alpha, \gamma) \in S^{\#}$ by two ways we obtain that

$$
n k \sum_{s \in S^{\#}} c(s)=n(k-1) k\left|S^{\#}\right|
$$

whence the required statement follows.

\subsection{Adjacency algebra}

Let $(\Omega, S)$ be a coherent configuration. The set $\{A(s): s \in S\}$ forms a linear basis of an algebra $\mathbb{C} S \subset \operatorname{Mat}_{\Omega}(\mathbb{C})$. It is called the adjacency algebra of the coherent configuration $(\Omega, S)$. From the definition it follows that it is closed with respect to the transpose and the Hadamard product. In particular, it is semisimple. So by the Wedderburn theorem its standard module $\mathbb{C} \Omega$ is completely reducible. For an irreducible submodule $L$ of $\mathbb{C} \Omega$ corresponding to a central primitive idempotent $P$ of the algebra $\mathbb{C} S$, we set

$$
n_{P}=\operatorname{dim}_{\mathbb{C}}(L), \quad m_{P}=\operatorname{rk}(P) / n_{P},
$$

thus $m_{P}$ and $n_{P}$ are the multiplicity and the degree of the corresponding irreducible representation of $\mathbb{C} S$. Clearly,

$$
|\Omega|=\sum_{P \in \mathcal{P}} m_{P} n_{P}, \quad|S|=\sum_{P \in \mathcal{P}} n_{P}^{2}
$$


where $\mathcal{P}$ is the set of central primitive idempotents of $\mathbb{C} S$. The coherent configuration $(\Omega, S)$ is called commutative if the algebra $\mathbb{C} S$ is commutative (equivalently, $n_{P}=1$ for all $P$ ).

Let $(\Omega, S)$ be a scheme. It can be proved that $m_{P} \geq n_{P}$ for all $P \in \mathcal{P}$ [10]. Besides, for the principal central primitive idempotent $P_{0}=\frac{1}{n} J$ where $J$ is the all one matrix, we have $m_{P_{0}}=n_{P_{0}}=1$. The corresponding one-dimensional irreducible representation of $\mathbb{C} S$ takes $A$ to $\operatorname{tr}\left(A A^{*}\right)$. In particular, we have

$$
n_{r} n_{s}=\sum_{t \in S} c_{r s}^{t} n_{t}
$$

We set $\mathcal{P}^{\#}=\mathcal{P} \backslash\left\{P_{0}\right\}$ and $\langle A, B\rangle=\operatorname{tr}\left(A B^{*}\right)$ for all $A, B \in \mathbb{C} S$.

\subsection{Intersection numbers}

There is a lot of useful identities for the intersection numbers of an arbitrary scheme [30]. One of them is (2.7), another one is

$$
n_{t} c_{r s}^{t^{*}}=n_{r} c_{s t}^{r^{*}}=n_{s} c_{t r}^{s^{*}}, \quad r, s, t \in S .
$$

We also need the following lemma.

Lemma 2.3. Let $(\Omega, S)$ be a scheme and $r, s \in S^{\#}$. Then $c_{r^{*} s}^{t} \leq 1$ for all $t \in S$ if and only if $r r^{*} \cap s s^{*}=\left\{1_{\Omega}\right\}$.

Proof. We have

$$
n_{r} n_{s} \leq n_{r} n_{s}+\sum_{t \in\left(r r^{*} \cap s s^{*}\right)^{\#}} c_{r r^{*}}^{t} c_{s s^{*}}^{t} n_{t}=\left\langle r r^{*}, s s^{*}\right\rangle
$$

and the bound is attained if and only if $r r^{*} \cap s s^{*}=\left\{1_{\Omega}\right\}$, where $\left\langle r r^{*}, s s^{*}\right\rangle=\left\langle A(r) A\left(r^{*}\right)\right.$, $\left.A(s) A\left(s^{*}\right)\right\rangle$. Similarly, from (2.7) it follows that

$$
\left\langle r^{*} s, r^{*} s\right\rangle=\sum_{t \in S}\left(c_{r^{*} s}^{t}\right)^{2} n_{t} \geq \sum_{t \in S} c_{r^{*} s}^{t} n_{t}=n_{r} n_{s}
$$

and the bound is attained if and only if $c_{r^{*} s}^{t} \leq 1$ for all $t \in S$. Since $\left\langle r r^{*}, s s^{*}\right\rangle=\left\langle r^{*} s, r^{*} s\right\rangle$ we are done.

\subsection{Frobenius groups}

In this subsection we recall some well-known group theoretical facts on the Frobenius groups [17, 27]. A non-regular transitive permutation group $G \leq \operatorname{Sym}(\Omega)$ is called a Frobenius group if $G_{\alpha, \beta}=\left\{\operatorname{id}_{\Omega}\right\}$ for all non-equal points $\alpha, \beta \in \Omega$. Any Frobenius group $G$ has a uniquely determined regular normal subgroup $A$ called the kernel of $G$. Therefore $G=A K$ where $K$ is a one point stabilizer of $G$, and $\operatorname{GCD}(|A|,|K|)=1$.

Theorem 2.4. Let $G \leq \operatorname{Sym}(\Omega)$ be a finite non-regular transitive permutation group with point stabilizer $K$ and

$$
\theta=\sum_{\chi \in \operatorname{Irr}(G)} m_{\chi} \chi
$$


the decomposition of the permutation character $\theta$ of $G$ into irreducibles. Then $G$ is a Frobenius group if and only if $\chi(1) / m_{\chi}=|K|$ for each character $\chi \in \operatorname{Irr}(G)^{\#}$ with $m_{\chi} \neq 0$.

Proof. To prove the necessity suppose that $G$ is a Frobenius group with a complement $K$. Then from [17, p.318-319] it follows that given $\varphi \in \operatorname{Irr}(K)^{\#}$ the class function $\chi_{\varphi}=$ $\varphi^{G}-\varphi(1) \theta^{\prime}$ is an irreducible character of $G$ of degree $\varphi(1)$ and

$$
\rho-|K| \theta^{\prime}=\mathbf{1}+\sum_{\varphi \in \operatorname{Irr}(K)^{\#}} \varphi(1) \chi_{\varphi}
$$

where 1 and $\rho$ are the principal and regular characters of $G$, and $\theta^{\prime}=\theta-1$. Since $\chi_{\varphi} \in \operatorname{Irr}(G)$, we have $\left[\rho, \chi_{\varphi}\right]=\chi_{\varphi}(1)=\varphi(1)$. Therefore by (2.9) we obtain

$$
\sum_{\chi \in \operatorname{Irr}(G) \backslash \Phi} \chi(1) \chi=|K| \theta^{\prime}=\sum_{\chi \in \operatorname{Irr}(G)^{\#}}|K| m_{\chi} \chi
$$

where $\Phi=\{\mathbf{1}\} \cup\left\{\chi_{\varphi}: \varphi \in \operatorname{Irr}(K)^{\#}\right\}$. Thus $\chi(1)=|K| m_{\chi}$ for each character $\chi \in \operatorname{Irr}(G)^{\#}$ with $m_{\chi} \neq 0$.

To prove the sufficiency suppose that $\chi(1) / m_{\chi}=|K|$ for each non-principal character $\chi \in \operatorname{Irr}(G)$ with $m_{\chi} \neq 0$. Since $\sum_{\chi} m_{\chi}^{2}=r-1$ where $r=|\operatorname{Orb}(K)|$ (see [17, Th. 16.6.14]), we have

$$
|\Omega|-1=\sum_{\chi \in \operatorname{Irr}(G)^{\#}} \chi(1) m_{\chi}=\sum_{\chi \in \operatorname{Irr}(G)^{\#}}|K| m_{\chi}^{2}=|K|(r-1) .
$$

Therefore $|K|=(|\Omega|-1) /(r-1)$. Since each non-trivial orbit of $K$ has cardinality at most $|K|$ and there are $r-1$ orbits, we obtain that each non-trivial $K$-orbit has cardinality $K$, that is $G_{\alpha, \beta}=\left\{\operatorname{id}_{\Omega}\right\}$ whenever $\alpha \neq \beta$.

\section{Pseudocyclic schemes}

A scheme $(\Omega, S)$ is called pseudocyclic if the number $m_{P} / n_{P}$ does not depend on the choice of the central primitive idempotent $P \in \mathcal{P}^{\#}$ (see Subsection 2.9). In the commutative case $n_{P}=1$ for all $P \in \mathcal{P}$, and our definition is compatible with that from [4]. Any regular scheme (not necessarily commutative) is pseudocyclic because in this case $m_{P}=n_{P}$ for all $P$. More elaborated example of a non-commutative pseudocyclic scheme arises from a Frobenius group with non-abelian kernel. In what follows we use the family of such groups given in [27, pp.187-189].

Example. Let $q$ be a prime power and $n>1$ an odd integer. Set $H$ to be a subgroup of $\mathrm{GL}\left(3, q^{n}\right)$ that consists of all matrices of the form

$$
A(a, b)=\left(\begin{array}{ccc}
1 & a & b \\
0 & 1 & a^{q} \\
0 & 0 & 1
\end{array}\right) \quad a, b \in \mathrm{GF}\left(q^{n}\right)
$$

Then the mapping $\sigma: A(a, b) \mapsto A\left(c a, c^{1+q} b\right)$ is a fixed point free automorphism of $H$ whenever the multiplicative order of $c$ equals $\left(q^{n}-1\right) /(q-1)$. So the semidirect 
product $G=H K$ where $K=\langle\sigma\rangle$, is a Frobenius group with non-abelian kernel $H$ and complement $K$. The natural action of $G$ on $H$ produces an equivalenced scheme of degree $|H|=q^{2 n}$, rank $q^{n+1}-q^{n}+q$ and valency $\left(q^{n}-1\right) /(q-1)$. A straightforward calculation for $(q, n)=(2,3)$ shows that the adjacency algebra of the corresponding scheme has exactly four irreducible characters, the multiplicities and degrees of which are as follows:

$$
\left(m_{1}, n_{1}\right)=(1,1),\left(m_{2}, n_{2}\right)=(7,1),\left(m_{3}, n_{3}\right)=\left(m_{4}, n_{4}\right)=(14,2) .
$$

In particular, the scheme is not commutative but is pseudocyclic because $m_{P} / n_{P}=7$ for all $P \in \mathcal{P}^{\#}$. In fact, this example is a special case of the following theorem.

Theorem 3.1. Any Frobenius scheme is pseudocyclic. Moreover, it is commutative if and only if the kernel of the associated Frobenius group is abelian.

Proof. Let $(\Omega, S)$ be a Frobenius scheme, $G \leq \operatorname{Sym}(\Omega)$ the corresponding Frobenius group and the mapping

$$
\pi: \mathbb{C} G \rightarrow \operatorname{Mat}_{\Omega}(\mathbb{C})
$$

is induced by the permutation representation of $G$ (in particular, $\pi(g)$ is the permutation matrix of a permutation $g \in G$ ). It is a well-known fact that a semisimple subalgebra of $\operatorname{Mat}_{\Omega}(\mathbb{C})$ coincides with the centralizer of its centralizer (in $\operatorname{Mat}_{\Omega}(\mathbb{C})$ ) [8, p.178]. Since the algebra $\pi(\mathbb{C} G)$ is semisimple and its centralizer in $\operatorname{Mat}_{\Omega}(\mathbb{C})$ equals $\mathbb{C} S$, the centralizer of $\mathbb{C} S$ in $\operatorname{Mat}_{\Omega}(\mathbb{C})$ coincides with $\pi(\mathbb{C} G)$. Therefore these two algebras have the same centre

$$
Z(\pi(\mathbb{C} G))=Z(\mathbb{C} S)=\mathbb{C} S \cap \pi(\mathbb{C} G),
$$

and hence the same set of central primitive idempotents, say $\mathcal{P}$. Moreover, one can see that $n_{P}=m_{\chi_{P}}$ and $m_{P}=\chi_{P}(1)$ for all $P \in \mathcal{P}$ where $\chi_{P}$ is the irreducible character of $G$ corresponding to the central primitive idempotent $P$. Since $G$ is a Frobenius group, by Theorem 2.4 this implies that

$$
m_{P} / n_{P}=m_{\chi_{P}} / \chi_{P}(1)=|K|, \quad P \in \mathcal{P}^{\#},
$$

where $K$ is the complement of $G$. Thus $(\Omega, S)$ is a pseudocyclic scheme.

To prove the second statement we find the dimension of the left-hand side of (3.1). First, we note that the algebra $Z(\mathbb{C} G)$ is spanned by the elements $C^{+}=\sum_{g \in C} g$ where $C$ runs through the set of conjugacy classes of $G$. Moreover, if $A$ denotes the kernel of $G$, then

$$
C \cap A=\emptyset \Rightarrow \pi\left(C^{+}\right) \in \mathbb{C} J_{\Omega} .
$$

(Indeed, $C$ contains $a^{-1} c a=a^{-1} a^{c} c$ for all $a \in A$ and $c \in C$. On the other hand, since $c \notin A$, the mapping $a \mapsto a^{-1} a^{c}, a \in A$, is a bijection. Thus $C A=C$. Taking into account that $\pi\left(A^{+}\right)=J_{\Omega}$, we conclude that $\pi\left(C^{+}\right)$is a scalar multiple of $J_{\Omega}$.) This implies that

$$
\operatorname{dim}(\pi(Z(\mathbb{C} G)))=\left|\operatorname{Cla}_{G}(A)\right|
$$

where $\operatorname{Cla}_{G}(A)$ is the set of conjugacy classes of $G$ contained in $A$. Indeed, $\pi\left(C^{+}\right)$is a $\{0,1\}$ matrix. Since the sum of these matrices with $C \in \mathrm{Cla}_{G}(A)$ equals $J_{\Omega}$, they are linearly independent and form a basis of $\pi(Z(\mathbb{C} G))$. 
Next, the group $K$ acts semiregularly on the set of non-trivial conjugacy classes of $A$. So any set $C \in \operatorname{Cla}_{G}(A)$ other than $\{1\}$ is a disjoint union of exactly $k=|K|$ of these classes. This shows that

$$
\left|\operatorname{Cla}_{G}(A)\right|=1+(|\operatorname{Cla}(A)|-1) / k
$$

where $\operatorname{Cla}(A)$ is the set of conjugacy classes of $A$. To complete the proof we note that from (3.1) it follows that the scheme $S$ is commutative if and only if $\mathbb{C} S=\pi(Z(\mathbb{C} G))$, or equivalently if

$$
1+(|A|-1) / k=|S|=\operatorname{dim}(\mathbb{C} S)=\operatorname{dim}(Z(\mathbb{C} S))=\operatorname{dim}(\pi(Z(\mathbb{C} G))) .
$$

By (3.2) and (3.3) this is true if and only if $|\mathrm{Cla}(A)|=|A|$, i.e. the group $A$ is commutative.

We would like to have a characterization of a pseudocyclic scheme in terms of its valences and indistiguishing numbers of basis relations. For commutative case it was done in [4, Proposition 2.2.7].

Theorem 3.2. The following two statements are equivalent:

(1) $(\Omega, S)$ is a pseudocyclic scheme with $m_{P} / n_{P}=k$ for all $P \in \mathcal{P}^{\#}$,

(2) $(\Omega, S)$ is an equivalenced scheme of valency $k$ with $c(s)=k-1$ for all $s \in S^{\#}$.

Moreover, any pseudocyclic scheme with pairwise equal non-principal dimensions of irreducible representations is commutative.

Proof. ${ }^{1}$ Denote by $\rho$ and by $\chi$ respectively the regular and standard characters of the algebra $\mathbb{C} S$. Then for any $s \in S$ we have

$$
\begin{gathered}
\rho\left(a_{s}\right)=\sum_{t \in S} c_{t s}^{t}=n_{s}+\sum_{P \in \mathcal{P}^{\#}} n_{P} \chi_{P}\left(a_{s}\right), \\
\chi\left(a_{s}\right)=\delta_{s, 1_{\Omega}} n=n_{s}+\sum_{P \in \mathcal{P}^{\#}}\left(m_{P} / n_{P}\right) n_{P} \chi_{P}\left(a_{s}\right)
\end{gathered}
$$

where $a_{s}=A(s), n=|\Omega|$ and $\chi_{P}$ is the irreducible character of of $\mathbb{C} S$ corresponding to central primitive idempotent $P$.

Suppose that $m_{P} / n_{P}=k$ for all $P \in \mathcal{P}^{\#}$. Then from (3.4) and (3.5) it follows that $k\left(n_{s}-\rho\left(a_{s}\right)\right)=n_{s}$ for all $s \in S^{\#}$. Since $n_{s}>0$, we have $n_{s}-\rho\left(a_{s}\right) \geq 1$, and hence $n_{s} \geq k$. By (2.6) this implies that

$$
n-1=\sum_{s \in S^{\#}} n_{s} \geq(r-1) k=\sum_{P \in \mathcal{P}^{\#}} n_{P} k=\sum_{P \in \mathcal{P}^{\#}} m_{P} n_{P}=n-1
$$

where $r=|S|$. Thus $n_{s}=k$ and $(\Omega, S)$ is an equivalenced scheme of valency $k$. In particular, $k\left(k-\rho\left(a_{s}\right)\right)=n_{s}$ whence it follows that, $\rho\left(a_{s}\right)=k-1$. However, by (3.4)

\footnotetext{
${ }^{1}$ The original proof was given in [24]. The authors thank to Paul-Hermann Zieschang who proposed a fruitful idea used in the proof here.
} 
and (2.8) we have $\rho\left(a_{s}\right)=c(s)$. Therefore $c(s)=k-1$ and the implication (1) $\Rightarrow(2)$ is proved.

Assume now that $n_{s}=k$ and $c(s)=k-1$ for all $s \in S^{\#}$. Then from (3.4) and (3.5) it follows that $k \leq k_{\text {min }}:=\min \left\{m_{P} / n_{P}: P \in \mathcal{P}^{\#}\right\}$. Therefore by (2.6) we have

$$
n-1=\sum_{P \in \mathcal{P}^{\#}} m_{P} n_{P}=\sum_{P \in \mathcal{P}^{\#}}\left(m_{P} / n_{P}\right) n_{P}^{2} \geq k_{\min } \sum_{P \in \mathcal{P}^{\#}} n_{P}^{2} \geq k(r-1)=n-1 .
$$

Thus $m_{P} / n_{P}=k_{m i n}=k$ for all $P \in \mathcal{P}^{\#}$. The implication $(2) \Rightarrow(1)$ is proved.

Let now $(\Omega, S)$ be a pseudocyclic scheme such that $n_{P}$ does not depend on $P \in \mathcal{P}^{\#}$. Denote this number by $a$. Then by the first part of the proof the scheme is equivalenced of valency $k$ where $k a=m_{P}$ for each $P \in \mathcal{P}^{\#}$. So from (2.6) it follows that $\left|\mathcal{P}^{\#}\right| a^{2}=$ $r-1=(n-1) / k$. Therefore $a$ is coprime to $n$. Taking into account that $n_{s}=k$ for all $s \in S^{\#}$, we have

$$
n^{r} \frac{\prod_{s \in S} n_{s}}{\prod_{P \in \mathcal{P}} m_{P}^{n_{P}^{2}}}=\frac{n^{r} k^{r-1}}{k^{r-1} a^{r-1}}=\frac{n^{r}}{a^{r-1}} .
$$

The number in the left-hand side, known as the Frame number [28] of the scheme $(\Omega, S)$, is an integer. Since $r>1$, we conclude that $a=1$. Thus $n_{P}=1$ for all $P$ and the scheme is commutative.

There is a lot of equivalenced schemes $(\Omega, S)$ for which the group of algebraic isomorphisms acts transitively on $S^{\#}$. These schemes were first studied by Ikuta, Ito and Munemasa [20], and include the cyclotomic schemes over finite fields and the schemes of affine planes (see Section 4). The following statement shows that all of them are pseudocyclic.

Corollary 3.3. Let $(\Omega, S)$ be an equivalenced scheme. Suppose that a group of its algebraic isomorphisms acts transitively on $S^{\#}$. Then $(\Omega, S)$ is a pseudocyclic scheme.

Proof. From the hypothesis it follows that the number $c(s)$ does not depend on $s \in S^{\#}$. So by Lemma 2.2 this number equals $k-1$ and we are done by Theorem 3.2.

Sometimes one can construct a new pseudocyclic scheme by means of an appropriate algebraic fusion defined as follows (see also [10, Lemma 3.1]). Let $G$ be a group of algebraic isomorphisms of a coherent configuration $(\Omega, S)$. Set

$$
S^{G}=\left\{s^{G}: s \in S\right\}
$$

where $s^{G}$ is the union of the relations $s^{g}, g \in G$. It is easily seen that the pair $\left(\Omega, S^{G}\right)$ is a coherent configuration. Moreover, if the group $G$ is half-transitive on $S^{\#}$ and the coherent configuration $(\Omega, S)$ is equivalenced, then the scheme $\left(\Omega, S^{G}\right)$ is equivalenced. An analog of this statement holds for commutative pseudocyclic schemes. It generalizes the results of Section 7.4 [22].

Theorem 3.4. Let $(\Omega, S)$ be a commutative pseudocyclic scheme of valency $k$ and $G a$ group of algebraic isomorphisms of it. Suppose that $G$ acts semiregularly on $S^{\#}$. Then $\left(\Omega, S^{G}\right)$ is a commutative pseudocyclic scheme of valency $\mathrm{km}$ where $m=|G|$. 
Proof. We observe that $\left(\Omega, S^{G}\right)$ being a fusion of a commutative scheme is also commutative. Since it is equivalenced of valency $\mathrm{km}$ (see above), we have

$$
\left|\mathcal{P}^{G}\right|=\left|S^{G}\right|=1+(|S|-1) / m
$$

where $\mathcal{P}^{G}$ is the set of central primitive idempotents of the algebra $\mathbb{C} S^{G}$. On the other hand, the group $G$ naturally acts as an automorphism group of the algebra $\mathbb{C} S$. Therefore the set $\mathcal{P}$ of its central primitive idempotents is $G$-invariant. For $P \in \mathcal{P}$ denote by $P^{G}$ the sum of all $Q \in \mathcal{P}$ belonging to the $G$-orbit containing $P$. Then obviously the set $\mathcal{P}^{\prime}=\left\{P^{G}: P \in \mathcal{P}\right\}$ consists of pairwise orthogonal central idempotents of the algebra $\mathbb{C} S^{G}$. This implies that

$$
\left|\mathcal{P}^{\prime}\right| \leq\left|\mathcal{P}^{G}\right|
$$

Finally, any $G$-orbit in $\mathcal{P}$ is of cardinality at most $m$. Since $G$ leaves $P_{0}$ fixed and $|\mathcal{P}|=|S|$, this implies that

$$
\left|\mathcal{P}^{\prime}\right| \geq 1+(|\mathcal{P}|-1) / m=1+(|S|-1) / m
$$

and the equality holds exactly when any $G$-orbit in $\mathcal{P}^{\#}$ is of size $m$. Together with (3.6) and (3.7) this shows that $\left|\mathcal{P}^{G}\right|=\left|\mathcal{P}^{\prime}\right|$. Therefore $m_{P} G=m m_{P}=m k$ and $n_{P}=1$ for all $P \in \mathcal{P}^{\#}$. Thus the scheme $\left(\Omega, S^{G}\right)$ is pseudocyclic.

A schurian equivalenced non-regular scheme is nothing but the scheme of $3 / 2$-transitive group. In general, the latter is not a Frobenius group. However, the following statement holds.

Theorem 3.5. A schurian pseudocyclic scheme of valency $k>1$ and rank greater than $2(k-1)$ is a Frobenius scheme the automorphism group of which is a Frobenius group.

Proof. Let $(\Omega, S)$ be a schurian pseudocyclic scheme of valency $k>1$ and let $G=$ $\operatorname{Aut}(\Omega, S)$. Then the set $\operatorname{Fix}(g)$ of points left fixed by a nonidentity permutation $g \in G$ does not coincide with $\Omega$. So there exists $\alpha \in \Omega$ such that $\alpha^{g} \neq \alpha$. Since $r(\alpha, \beta)=$ $r\left(\alpha^{g}, \beta\right)$ for all $\beta \in \operatorname{Fix}(g)$, Theorem 3.2 implies that

$$
|\operatorname{Fix}(g)| \leq\left|\left\{\beta \in \Omega: r(\alpha, \beta)=r\left(\alpha^{g}, \beta\right)\right\}\right|=c(s)=k-1
$$

where $s=r\left(\alpha, \alpha^{g}\right)$. Thus the permutation character of $G$ takes the value in the set $\{0, \ldots, k-1\}$ on all nonidentity elements. Therefore by [6, Prop.1] a point stabilizer $G_{\alpha}$ has at most $2(k-1)-1$ non-regular orbits. If $|S|>2(k-1)$, then at least one orbit of $G_{\alpha}$ is regular. This implies that so are all non-trivial orbits. Thus $G$ is a Frobenius group.

\section{Known examples of pseudocyclic schemes}

\subsection{Schemes of rank 3}

Any pseudocyclic scheme of rank 3 arises from either a conference matrix (symmetric case) or from a skew Hadamard matrix (antisymmetric case) [4, 25]. In any case the intersection number array is uniquely determined by the degree of the scheme. In particular, two such schemes are algebraically isomorphic if and only if they have the same degree. Since there is an infinite number of $n$ for which there are at least two non-equivalent conference matrices or non-equivalent skew Hadamard matrices of order $n$, in general pseudocyclic 
schemes of rank 3 are not separable (see also [12, Section 7]). Similarly, one can see that most of them are non-schurian. For instance, it follows from [3] that an antisymmetric pseudocyclic scheme of rank 3 is schurian if and only if it is a scheme of a Paley tournament. Moreover, in [15, p.75] one can find an infinite family of non-schurian pseudocyclic schemes of rank 3 satisfying the 4-condition.

\subsection{The Hollman schemes [4, p.390]}

Let $q>4$ be a power of 2 . Denote by $\Omega$ the set of cyclic groups of order $q+1$ in the group $\operatorname{PSL}(2, q)$. The latter acts transitively on $\Omega$ by conjugation and hence produces the scheme of degree $\left(q^{2}-q\right) / 2$. One can prove that this scheme is symmetric and pseudocyclic of valency $q+1$. Some algebraic fusions of the Hollman scheme that are also pseudocyclic were studied in [19].

\subsection{The Passman schemes [26]}

Let $q$ be an odd prime power and $F$ the group consisting of the transformations

$$
\left(\begin{array}{l}
x \\
y
\end{array}\right) \rightarrow\left(\begin{array}{cc}
a & 0 \\
0 & a^{-1}
\end{array}\right)\left(\begin{array}{l}
x \\
y
\end{array}\right)+\left(\begin{array}{l}
b \\
c
\end{array}\right),
$$

where $a, b, c \in \operatorname{GF}(q)$, and $a \neq 0$. Then $F$ is a Frobenius group acting on a 2-dimensional space over $\operatorname{GF}(q)$. By Theorem 3.1 the scheme of $F$ is pseudocyclic of valency $q-1$. The group

$$
D=\left\{\left(\begin{array}{cc} 
\pm 1 & 0 \\
0 & \pm 1
\end{array}\right),\left(\begin{array}{cc}
0 & \pm 1 \\
\pm 1 & 0
\end{array}\right)\right\}
$$

normalizes $F$ and, therefore, acts on the relations of the scheme of $F$ as a group of algebraic automorphisms. A direct check shows that the action of $D$ on non-trivial relations of the scheme of $F$ is semiregular with orbit length two. By Theorem 3.4 the scheme of the group $F D$ pseudocyclic of valency $2(q-1)$. We call the scheme of the group $F D$ the Passman scheme, because Passman pointed out that $F D$ is 3/2-transitive non-Frobenius solvable group.

\subsection{Cyclotomic schemes}

Let $R$ be a finite local commutative ring with identity. Then its multiplicative group $R^{\times}$is the direct product of the Teichmüller group $\mathcal{T}$ and the group of principal units [23]. The Teichmüller group is isomorphic to the multiplicative group of the residue field of $R$, and acts as a fixed point free automorphism group of the additive group $R^{+}$of $R$. Therefore for a given $K \leq \mathcal{T}$, the scheme of the group $\left(R^{+} \rtimes K, R^{+}\right)$is a Frobenius scheme and hence pseudocyclic by Theorem 3.1. This example is a special case of a cyclotomic scheme over a finite commutative ring [14]. In almost the same way one can construct a class of pseudocyclic schemes where the ground ring $R$ is replaced by a near-field [2], or even a near-ring.

\subsection{Affine schemes}

Let $\Omega$ be the point set of a finite affine space $\mathcal{A}$ (see [5]). Denote by $S$ the partition of $\Omega \times \Omega$ containing $1_{\Omega}$ and such that two pairs $(\alpha, \beta),\left(\alpha^{\prime}, \beta^{\prime}\right) \in \Omega \times \Omega, \alpha \neq \beta, \alpha^{\prime} \neq \beta^{\prime}$, belong 
to the same class if and only if the lines $\alpha \beta$ and $\alpha^{\prime} \beta^{\prime}$ are equal or parallel. Then the pair $(\Omega, S)$ is a symmetric scheme and nonzero intersection numbers $c_{r s}^{t}$ with $1_{\Omega} \notin\{r, s\}$ are as follows:

$$
c_{r s}^{t}= \begin{cases}q-1, & r=s, t=1_{\Omega}, \\ q-2, & r=s=t, \\ 1, & r \neq s, t \in r s\end{cases}
$$

where $q$ is the size of a line in $\mathcal{A}$ (called the order of $\mathcal{A}$ ). It follows that it is a pseudocyclic scheme of valency $q-1$. Each relation of an affine scheme is an involution in the sense of [30]. It was shown in [9] that a scheme whose relations are involutions is an affine scheme. Thus there is one-to-one correspondence between affine schemes and affine spaces. It is straightforward to prove that the schemes of affine spaces are isomorphic (resp. algebraically isomorphic) if and only if the affine spaces are isomorphic (resp. have the same order).

Theorem 4.1. The scheme of a finite affine space $\mathcal{A}$ is schurian if and only if $\mathcal{A}$ is Desarguesian.

Proof. By the Veblen-Young theorem (cf. [5]) a finite affine space $\mathcal{A}$ is either a nonDesarguesian affine plane, or the $n$-dimensional affine geometry $\operatorname{AG}(n, q)$ over $\operatorname{GF}(q)$. In the latter case the scheme of $\mathcal{A}$ coincides with the scheme of the group

$$
T C \leq \operatorname{AGL}(n, q)
$$

where $T$ is the translation group and $C$ is the centre of $\operatorname{GL}(n, q)$. This proves the sufficiency part of the theorem. To prove the necessity assume that the scheme of $\mathcal{A}$ is schurian. Then it satisfies the 4-condition and the required statement immediately follows from the lemma below.

Lemma 4.2. Suppose that the scheme of an affine space $\mathcal{A}$ satisfies the 4-condition. Then $\mathcal{A}$ is Desarguesian.

Proof. Let $(\Omega, S)$ be the scheme of $\mathcal{A}$. It suffices to verify that given seven distinct points $\alpha, \alpha^{\prime}, \beta, \beta^{\prime}, \gamma, \gamma^{\prime}$ and $\delta$, such that $\alpha \alpha^{\prime}, \beta \beta^{\prime}$, and $\gamma \gamma^{\prime}$ are distinct lines through $\delta$ and $\alpha \gamma$ is parallel to $\alpha^{\prime} \gamma^{\prime}$ and $\beta \gamma$ is parallel to $\beta^{\prime} \gamma^{\prime}$, then $\alpha \beta$ is parallel to $\alpha^{\prime} \beta^{\prime}$ (see Figure 1). However, since $\delta \gamma=\delta \gamma^{\prime}$, we have $r(\delta, \gamma)=r\left(\delta, \gamma^{\prime}\right)$. Due to the 4-condition there exist points $\alpha^{\prime \prime}, \beta^{\prime \prime}$ such that the 4 -sets $\Delta=\{\delta, \gamma, \alpha, \beta\}$ and $\Delta^{\prime}=\left\{\delta, \gamma^{\prime}, \alpha^{\prime \prime}, \beta^{\prime \prime}\right\}$ have the same type with respect to the pairs $(\delta, \gamma)$ and $\left(\delta, \gamma^{\prime}\right)$ respectively. So $r(\alpha, \delta)=r\left(\alpha^{\prime \prime}, \delta\right)$ and $r(\beta, \delta)=r\left(\beta^{\prime \prime}, \delta\right)$. This implies that

$$
\alpha^{\prime \prime} \in \alpha \delta=\alpha^{\prime} \delta, \quad \beta^{\prime \prime} \in \beta \delta=\beta^{\prime} \delta .
$$

On the other hand, $r(\alpha, \gamma)=r\left(\alpha^{\prime \prime}, \gamma^{\prime}\right)$ and $r(\beta, \gamma)=r\left(\beta^{\prime \prime}, \gamma^{\prime}\right)$. Therefore $\alpha \gamma$ is parallel to $\alpha^{\prime \prime} \gamma^{\prime}$, and $\beta \gamma$ is parallel to $\beta^{\prime \prime} \gamma^{\prime}$. Thus from (4.4) we conclude that $\alpha^{\prime \prime}=\alpha^{\prime}$ and $\beta^{\prime \prime}=\beta^{\prime}$. Since also $r(\alpha, \beta)=r\left(\alpha^{\prime \prime}, \beta^{\prime \prime}\right)$, the line $\alpha \beta$ is parallel to $\alpha^{\prime \prime} \beta^{\prime \prime}=\alpha^{\prime} \beta^{\prime}$, and we are done. 


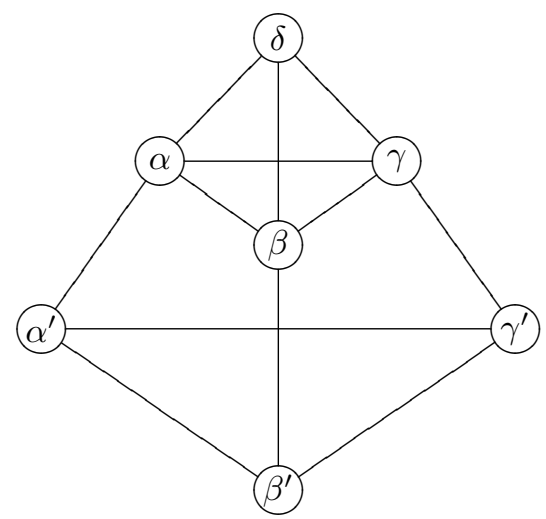

Figure 1:

\subsection{Amorphic schemes}

A scheme $(\Omega, S)$ is called amorphic [16] if any its fusion is a scheme. It was shown in [16] that all basis graphs of an amorphic scheme of rank at least four are strongly regular either of Latin square type or of negative Latin square type. If an amorphic scheme is equivalenced, then its group of algebraic automorphisms is $\operatorname{Sym}\left(S^{\#}\right)$. This implies (Corollary 3.3) that $(\Omega, S)$ is pseudocyclic. A scheme of an affine plane of order $q$ is an amorphic $(q-1)$-valenced scheme of rank $q+2$. This yields us the following statement.

Theorem 4.3. Let $q$ be the order of an affine plane. Then given a divisor $m$ of $q+1$ and a partition of $\{1, \ldots, q+1\}$ in $m$ classes of cardinality $(q+1) / m$, there exists an amorphic pseudocyclic scheme of degree $q^{2}$, valency $\left(q^{2}-1\right) / m$ and rank $m+1$.

\section{One point fission of an equivalenced scheme.}

\subsection{Splitting sets}

Let $(\Omega, S)$ be an equivalenced scheme of valency $k$. For each (possibly equal) basis relations $u, v \in S^{\#}$ we define the splitting set $D(u, v)$ of them as follows:

$$
D(u, v)=\left\{w \in S^{\#}:\left(u u^{*} v v^{*}\right) \cap w w^{*}=\left\{1_{\Omega}\right\}\right\} .
$$

It is easily seen that $D(u, v)=D(v, u)$ and $u u^{*} \cap w w^{*}=v v^{*} \cap w w^{*}=\left\{1_{\Omega}\right\}$ for all $w \in D(u, v)$. Therefore from Lemma 2.3 it follows that

$$
c_{u^{*} w}^{s} \leq 1 \quad \text { and } \quad c_{w^{*} v}^{s} \leq 1
$$

for all $s \in S$. In particular, $\left|u^{*} w\right|=\left|w^{*} v\right|=k$.

Theorem 5.1. Given $w \in D(u, v)$ the following statements hold:

(1) $\left|u^{*} v\right|=k \Leftrightarrow u \in D(v, w) \Leftrightarrow v \in D(w, u)$,

(2) $\left|a b \cap u^{*} v\right|=1$ for all $a \in u^{*} w$ and $b \in w^{*} v$. 
Proof. To prove statement (1) suppose that $\left|u^{*} v\right|=k$. Then $c_{u^{*} v}^{t} \leq 1$ for all $t \in S$. So from Lemma 2.3 it follows that $u u^{*} \cap v v^{*}=\left\{1_{\Omega}\right\}$. On the other hand, given a relation $s \in\left(v v^{*} w w^{*}\right) \cap u u^{*}$ one can find points $\alpha, \beta \in \Omega$ to have the configuration at Fig. 2. So $r(\alpha, \beta) \in\left(u u^{*} v v^{*}\right) \cap w w^{*}=\left\{1_{\Omega}\right\}$ whence it follows that $\alpha=\beta$. There-

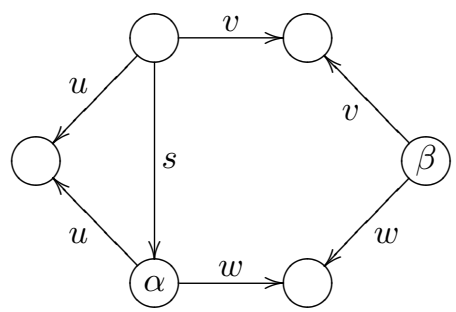

Figure 2:

fore $s \in v v^{*} \cap u u^{*}=\left\{1_{\Omega}\right\}$. Thus $s=1_{\Omega}$, and hence $u \in D(v, w)$. Conversely, if $u \in D(v, w)$, then $\left(v v^{*} w w^{*}\right) \cap u u^{*}=\left\{1_{\Omega}\right\}$. So $v v^{*} \cap u u^{*}=\left\{1_{\Omega}\right\}$, and hence $\left|u^{*} v\right|=k$ by Lemma 2.3. This completes the proof of the first equivalence in statement (1). The second equivalence immediately follows from the first one by interchanging $u$ and $v$ because $D(u, v)=D(v, u)$.

To prove statement (2) let $a \in u^{*} w$ and $b \in w^{*} v$. Then $w \in u a \cap v b^{*}$. This implies that $\left|u a \cap v b^{*}\right| \geq 1$, and hence $\left|a b \cap u^{*} v\right| \geq 1$. Thus it suffices to verify that $\left|a b \cap u^{*} v\right| \leq 1$. To do this we need the following auxiliary statement.

Lemma 5.2. Given a relation $s \in a b \cap u^{*} v$ and points $\alpha, \beta, \gamma \in \Omega$ such that $r(\alpha, \beta)=s$, $r(\alpha, \gamma)=a$ and $r(\gamma, \beta)=b$ there exists a unique point $\delta \in \Omega$ for which $r(\delta, \alpha)=u$, $r(\delta, \beta)=v$ and $r(\delta, \gamma)=w($ see Fig. 3).

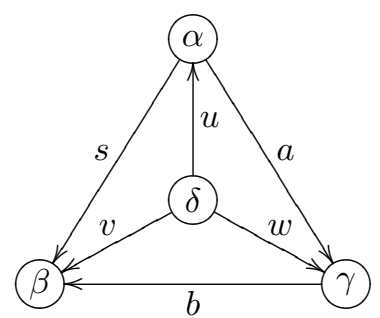

Figure 3:

Proof. Since $a \in u^{*} w$ and $b \in w^{*} v$, there exist points $\lambda, \mu, \nu$ such that $r(\lambda, \alpha)=u$, $r(\lambda, \beta)=v, r(\mu, \alpha)=u, r(\mu, \gamma)=w$ and $r(\nu, \gamma)=w, r(\nu, \beta)=v$ (see Fig. 4). Now $r(\mu, \nu) \in\left(u u^{*} v v^{*}\right) \cap w w^{*}=\left\{1_{\Omega}\right\}$. Thus $\mu=\nu$. Denote this point by $\delta$. Then for $\delta$ the statement of the lemma holds. To prove the uniqueness we note if $\delta_{1}$ and $\delta_{2}$ are two points forming Fig. 3, then the relation $r\left(\delta_{1}, \delta_{2}\right)$ belongs to the set $w w^{*} \cap u u^{*} \cap v v^{*}=\left\{1_{\Omega}\right\}$, and hence $\delta_{1}=\delta_{2}$. 


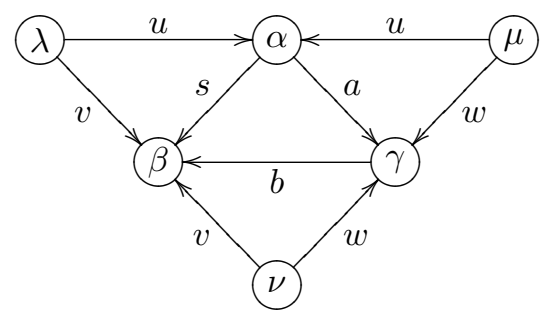

Figure 4:

To complete the proof of Theorem 5.1 suppose that $a b \cap u^{*} v \supset\left\{s_{1}, s_{2}\right\}$ with $s_{1} \neq s_{2}$. Then there exist points $\alpha, \gamma, \beta_{1}, \beta_{2} \in \Omega$, such that $\beta_{1} \neq \beta_{2}, r(\alpha, \gamma)=a$ and $r\left(\gamma, \beta_{i}\right)=b$, $r\left(\alpha, \beta_{i}\right)=s_{i}$ for $i=1,2$. By Lemma 5.2 with $s=s_{i}$ one can find a point $\delta_{i}$ for which $r\left(\delta_{i}, \alpha\right)=u, r\left(\delta_{i}, \beta\right)=v$ and $r\left(\delta_{i}, \gamma\right)=w$ (see Fig. 5). Since the relation $r\left(\delta_{1}, \delta_{2}\right)$

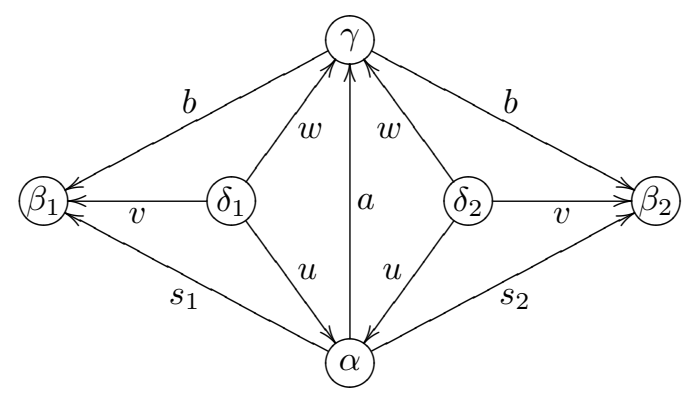

Figure 5:

belongs to the set $w w^{*} \cap u u^{*}=\left\{1_{\Omega}\right\}$, we have $\delta_{1}=\delta_{2}$. Denote this point by $\delta$. Then $r\left(\delta, \beta_{i}\right)=v$. Since $r\left(\beta_{i}, \gamma\right)=b^{*}, r(\delta, \gamma)=w$ and $\beta_{1} \neq \beta_{2}$, this implies that $c_{v b^{*}}^{w} \geq$ 2. So by equivalencity $(X, \Omega)$ and (2.8) we conclude that $c_{w^{*} v}^{b} \geq 2$ which contradicts to (5.2).

\subsection{A one point fission}

By means of splitting sets we are going to find explicitly the $\alpha_{0}$-fission of the scheme $(\Omega, S)$ for any point $\alpha_{0} \in \Omega$. To do this for any relations $u, v \in S$ set

$$
S(u, v)=S_{\alpha_{0}}(u, v)=\left\{s \cap\left(\alpha_{0} u \times \alpha_{0} v\right): s \in u^{*} v\right\} .
$$

It is easily seen that the union of relations from $S(u, v)$ coincides with the set $\alpha_{0} u \times \alpha_{0} v$. Suppose that $w \in D(u, v)$. Then from statement (2) of Theorem 5.1 it follows that

$$
S(u, v) \subset S(u, v ; w)^{\cup}
$$

where $S(u, v ; w)=S_{\alpha_{0}}(u, v ; w)=S(u, w) \cdot S(w, v)$. Suppose, in addition, that $\left|u^{*} v\right|=$ $k$. Then any relation in $S(u, v)$ is of cardinality $k$. Since the same is true for the relations in $S(u, v ; w)$, we conclude that

$$
\left(w \in D(u, v) \quad \& \quad\left|u^{*} v\right|=k\right) \Rightarrow S(u, v)=S(u, v ; w) .
$$


In particular, in this case the relations from $S(u, v ; w)$ form a partition of the set $\alpha_{0} u \times$ $\alpha_{0} v$. For arbitrary $u$ and $v$ we will prove the latter only under the following additional assumption:

$$
\bigcap_{\substack{a \in\{u, v\} \\ b \in\left\{w, w^{\prime}\right\}}} D(a, b) \neq \emptyset, \quad w, w^{\prime} \in D(u, v) .
$$

In this case obviously $D(u, v) \neq \emptyset$.

Lemma 5.3. Let $u, v \in S^{\#}$ be such that (5.6) holds. Then the set $S(u, v ; w)$ forms $a$ partition of the set $\alpha_{0} u \times \alpha_{0} v$, and this partition does not depend on the choice of the relation $w \in D(u, v)$.

Proof. Let $w, w^{\prime} \in D(u, v)$. Then $\left|u^{*} w\right|=\left|u^{*} w^{\prime}\right|=\left|v^{*} w\right|=\left|v^{*} w^{\prime}\right|=k$. On the other hand, due to (5.6) one can find a relation

$$
t \in D(u, w) \cap D\left(u, w^{\prime}\right) \cap D(v, w) \cap D\left(v, w^{\prime}\right) .
$$

So from (5.5) it follows that

$$
S(x, y)=S(x, y ; t)=S(x, t) \cdot S(t, y), \quad x \in\{u, v\}, y \in\left\{w, w^{\prime}\right\} .
$$

Let $a \in S(u, t)$ and $b \in S(t, v)$. Since obviously $b^{*} \in S(v, t)$ by (5.7) with $(x, y)=(u, w)$ and $(x, y)=(v, w)$ we obtain

$$
a \cdot c \in S(u, w), \quad b^{*} \cdot c \in S(v, w), \quad c \in S(t, w) .
$$

So the element $a \cdot b \in S(u, v ; w)$ has at least $k$ different representations (one for each choice of $c)$ of the form $a \cdot b=(a \cdot c)\left(c^{*} \cdot b\right)$ with $c^{*} \cdot b \in S(w, v)$. Since $|S(u, w)|=$ $|S(w, v)|=k$ this implies that $|S(u, v ; w)|=k$. Thus $S(u, v ; w)$ is a partition of $\alpha_{0} u \times$ $\alpha_{0} v$ and $S(u, v ; w)=S(u, v ; t)$. Similarly, using equalities (5.7) with $(x, y)=\left(u, w^{\prime}\right)$ and $(x, y)=\left(v, w^{\prime}\right)$ one can prove that $S\left(u, v ; w^{\prime}\right)$ is a partition of $\alpha_{0} u \times \alpha_{0} v$ and that $S\left(u, v ; w^{\prime}\right)=S(u, v ; t)$. Thus

$$
S(u, v ; w)=S(u, v ; t)=S\left(u, v ; w^{\prime}\right)
$$

and we are done.

By Lemma 5.3 we can define a uniquely determined partition of the set $\Omega_{0} \times \Omega_{0}$ where $\Omega_{0}=\Omega \backslash\left\{\alpha_{0}\right\}$, as follows

$$
S_{0}=S_{0}\left(\alpha_{0}\right)=\bigcup_{w \in D(u, v)} S_{\alpha_{0}}(u, v ; w) .
$$

It is easily seen that $1_{\alpha_{0} u} \in S_{0}$ for all $u \in S^{\#}$. Therefore $1_{\Omega_{0}} \in S_{0}^{\cup}$. Besides, since obviously $S(u, v ; w)^{*}=S(v, u ; w)$ for all $u, v, w$, the partition $S_{0}$ is closed with respect to $*$. Finally, the condition (2.2) is satisfied for $S=S_{0}$. Therefore if $\left(\Omega_{0}, S_{0}\right)$ is a coherent configuration, then it is semiregular. We will prove the former under the following assumption:

$$
D(u, v) \cap D(v, w) \cap D(w, u) \neq \emptyset
$$

for all $u, v, w \in S^{\#}$. Below we set

$$
S_{1}\left(\alpha_{0}\right)=\left\{\left\{\alpha_{0}\right\} \times \alpha_{0} v: v \in S\right\} \cup\left\{\alpha_{0} v \times\left\{\alpha_{0}\right\}: v \in S\right\} .
$$


Theorem 5.4. Let $(\Omega, S)$ be an equivalenced scheme satisfying conditions (5.6) and (5.9) for all $u, v \in S^{\#}$. Then

$$
S_{\alpha_{0}}=S_{0}\left(\alpha_{0}\right) \cup S_{1}\left(\alpha_{0}\right), \quad \alpha_{0} \in \Omega .
$$

In particular, the $\alpha_{0}$-fission of $(\Omega, S)$ is a semiregular coherent configuration on $\Omega_{0}$ the fibers of which are $\alpha_{0} u, u \in S$.

Proof. It suffices to verify that $\left(\Omega_{0}, S_{0}\right)$ is a coherent configuration. Indeed, if it is so and $S^{\prime}=S_{0}\left(\alpha_{0}\right) \cup S_{1}\left(\alpha_{0}\right)$, then obviously $\left(\Omega, S^{\prime}\right)$ is a semiregular coherent configuration the fibers of which are $\alpha_{0} u, u \in S$. Therefore due to (5.4) we have $1_{\alpha_{0}} \in S^{\prime}$ and $S \subset\left(S^{\prime}\right)^{\cup}$. By the minimality of the $\alpha_{0}$-fission this implies that

$$
S_{\alpha_{0}} \subset\left(S^{\prime}\right)^{\cup}
$$

On the other hand, it is easily seen that the set $S(u, v)$, and hence the set $S(u, v ; w)=$ $S(u, w) \cdot S(w, v)$ is contained in $\left(S_{\alpha_{0}}\right)^{\cup}$ for all $u, v, w \in S$. Therefore $S^{\prime} \subset\left(S_{\alpha_{0}}\right)^{\cup}$. Together with (5.10) this shows that $\left(S_{\alpha_{0}}\right)^{\cup}=\left(S^{\prime}\right)^{\cup}$. Thus $S_{\alpha_{0}}=S^{\prime}$ and we are done.

Let us prove that $\left(\Omega_{0}, S_{0}\right)$ is a coherent configuration. We observe that due to (5.6) Lemma 5.3 implies that $S_{0}$ is a partition of $\Omega_{0} \times \Omega_{0}$. Thus it suffices to verify that if $b, c \in S_{0}$ with $b \cdot c \neq \emptyset$, then $b \cdot c \in S_{0}$. However, for such $b$ and $c$ we have

$$
b \subset \alpha_{0} u \times \alpha_{0} v, \quad c \subset \alpha_{0} v \times \alpha_{0} w
$$

for appropriate $u, v, w \in S^{\#}$. By condition (5.9) one can find

$$
t \in D(u, v) \cap D(v, w) \cap D(w, u) .
$$

Since $b=a_{1} \cdot a_{2}$ for some $a_{1} \in S(u, v ; t)$ and $a_{2} \in S(v, w ; t)$, this implies that $|S(v, t)|=$ $|S(w, t)|=k$ where $k$ is the valency of $(\Omega, S)$. So by statement (1) of Theorem 5.1 we have $w \in D(v, t)$. Therefore $a_{2}^{*} \in S(v, t ; w)$ and hence there exists $a_{3} \in S(t, w)$ such that $c=a_{2}^{*} \cdot a_{3}$. Thus

$$
b \cdot c=\left(a_{1} \cdot a_{2}\right) \cdot\left(a_{2}^{*} \cdot a_{3}\right)=a_{1} \cdot\left(a_{2} \cdot a_{2}^{*}\right) \cdot a_{3} \in S(u, w ; t) .
$$

This means that $b \cdot c \in S_{0}$ which completes the proof.

\section{One point extension of an algebraic isomorphism}

We keep the notation of Section 5. Let $\left(\Omega^{\prime}, S^{\prime}\right)$ be a scheme and let

$$
\varphi:(\Omega, S) \rightarrow\left(\Omega^{\prime}, S^{\prime}\right), s \mapsto s^{\prime}
$$

be an algebraic isomorphism. Then obviously $\left(\Omega^{\prime}, S^{\prime}\right)$ is an equivalenced scheme of valency $k$, and

$$
w \in D(u, v) \Leftrightarrow w^{\prime} \in D\left(u^{\prime}, v^{\prime}\right), \quad u, v \in S^{\#} .
$$

Let us fix a point $\alpha_{0}^{\prime} \in \Omega^{\prime}$. Take $u, v \in S$. Since $c_{u v}^{w}=c_{u^{\prime} v^{\prime}}^{w^{\prime}}$ for all $w \in S$, the mapping $\varphi$ induces a bijection

$$
\varphi_{u, v}: S_{\alpha_{0}}(u, v) \rightarrow S_{\alpha_{0}^{\prime}}\left(u^{\prime}, v^{\prime}\right), \quad a \mapsto a^{\prime}
$$

where $a=s \cap\left(\alpha_{0} u \times \alpha_{0} v\right)$ and $a^{\prime}=s^{\prime} \cap\left(\alpha_{0}^{\prime} u^{\prime} \times \alpha_{0}^{\prime} v^{\prime}\right)$. Below we set $S^{\prime}\left(u^{\prime}, v^{\prime}\right)=$ $S_{\alpha_{0}^{\prime}}\left(u^{\prime}, v^{\prime}\right)$. 
Lemma 6.1. Let $u, v \in S^{\#}$ be such that (5.6) holds. Then for any relations $w_{1}, w_{2} \in$ $D(u, v)$ we have

$$
b_{1} \cdot c_{1}=b_{2} \cdot c_{2} \Rightarrow b_{1}^{\prime} \cdot c_{1}^{\prime}=b_{2}^{\prime} \cdot c_{2}^{\prime}
$$

for all $b_{i} \in S\left(u, w_{i}\right)$ and $c_{i} \in S\left(w_{i}, v\right), i=1,2$.

Proof. Suppose that $b_{1} \cdot c_{1}=b_{2} \cdot c_{2}$ where $b_{i} \in S\left(u, w_{i}\right)$ and $c_{i} \in S\left(w_{i}, v\right), i=1,2$. Then $\left|u^{*} w_{1}\right|=\left|u^{*} w_{2}\right|=\left|v^{*} w_{1}\right|=\left|v^{*} w_{2}\right|=k$. Besides, by (5.6) one can find a relation

$$
t \in D\left(u, w_{1}\right) \cap D\left(u, w_{2}\right) \cap D\left(v, w_{1}\right) \cap D\left(v, w_{2}\right) .
$$

So from (5.5) it follows that

$$
S(x, y ; t)=S(x, t) \cdot S(t, y), \quad x \in\{u, v\}, y \in\left\{w_{1}, w_{2}\right\} .
$$

Take any $a_{1} \in S(u, t)$. By (6.1) we have $d_{1}:=a_{1}^{*} b_{1} \in S\left(t, w_{1}\right)$ and $d_{2}:=a_{1}^{*} \cdot b_{2} \in$ $S\left(t, w_{2}\right)$. Since $w_{1} \in D(t, v)$, we also have $a_{2}:=d_{1} \cdot c_{1} \in S(t, v)$. Thus

$$
a_{1} \cdot a_{2}=\left(a_{1} \cdot d_{1}\right) \cdot\left(d_{1}^{*} \cdot a_{2}\right)=b_{1} \cdot c_{1}=b_{2} \cdot c_{2}=a_{1} \cdot d_{2} \cdot c_{2}
$$

whence it follows that $c_{2}=d_{2}^{*} \cdot a_{2}$ (Figure 6). By Theorem 5.1 this implies that

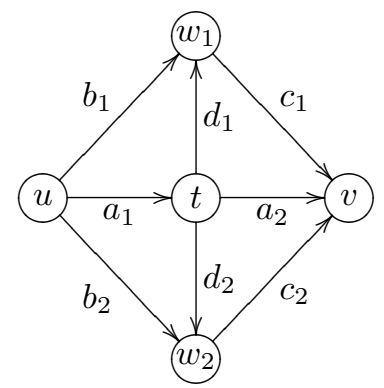

Figure 6:

$$
\begin{gathered}
b_{1}^{\prime} \cdot c_{1}^{\prime}=\left(a_{1} \cdot d_{1}\right)^{\prime} \cdot\left(d_{1}^{*} \cdot a_{2}\right)^{\prime}=a_{1}^{\prime} \cdot\left(d_{1}^{\prime} \cdot\left(d_{1}^{\prime}\right)^{*}\right) \cdot a_{2}^{\prime}=a_{1}^{\prime} \cdot a_{2}^{\prime}= \\
\left(b_{2} \cdot d_{2}^{*}\right)^{\prime} \cdot\left(d_{2} \cdot c_{2}\right)^{\prime}=b_{2}^{\prime} \cdot\left(d_{2}^{\prime} \cdot\left(d_{2}^{*}\right)^{\prime}\right) \cdot c_{2}^{\prime}=b_{2}^{\prime} \cdot c_{2}^{\prime}
\end{gathered}
$$

and we are done.

Let us define a mapping $\varphi_{0}: S_{0} \rightarrow S_{0}^{\prime}$ where $S_{0}=S_{\alpha_{0}}$ and $S_{0}^{\prime}=S_{\alpha_{0}^{\prime}}^{\prime}$, as follows. Take $s \in S_{0}$. Then $s \subset \alpha_{0} u \times \alpha_{0} v$ for some $u, v \in S$. Set

$$
s^{\varphi_{0}}= \begin{cases}s^{\varphi_{u, v}}, & \text { if }\left|u^{*} v\right|=k \text { or } 1_{\Omega} \in\{u, v\} \\ s_{1}^{\varphi_{u, w}} \cdot s_{2}^{\varphi_{w, v}}, & \text { otherwise }\end{cases}
$$

where $w \in D(u, v)$ and $s_{1} \in S(u, w), s_{2} \in S(w, v)$ are such that $s_{1} \cdot s_{2}=s$. By Theorem 5.4 and Lemma 6.1 the mapping $\varphi_{0}$ is a correctly defined bijection. 
Theorem 6.2. Let $(\Omega, S)$ be an equivalenced scheme satisfying conditions (5.6) and (5.9) for all $u, v \in S^{\#}$, and $\varphi:(\Omega, S) \rightarrow\left(\Omega^{\prime}, S^{\prime}\right)$ an algebraic isomorphism. Then $\varphi_{0}$ is the $\left(\alpha_{0}, \alpha_{0}^{\prime}\right)$-extension of $\varphi$.

Proof. We observe that relations (2.4) hold by statement (2) of Theorem 5.1. Therefore it suffices to verify that

$$
(b \cdot c)^{\varphi_{0}}=b^{\varphi_{0}} \cdot c^{\varphi_{0}}, \quad b, c \in S_{0}, b \cdot c \neq \emptyset .
$$

To do this take such relations $b$ and $c$. Then there exist $u, v, w \in S^{\#}$ such that

$$
b \subset \alpha_{0} u \times \alpha_{0} v, \quad c \subset \alpha_{0} v \times \alpha_{0} w .
$$

By condition (5.9) one can find $t \in D(u, v) \cap D(v, w) \cap D(w, u)$. Since $b \in S_{0}$, there exist $a_{1} \in S(u, t)$ and $a_{2} \in S(t, v)$ such that $b=a_{1} \cdot a_{2}$. However, $|S(u, t)|=|S(t, v)|=$ $k$. Therefore $b^{\varphi_{0}}=a_{1}^{\varphi_{0}} \cdot a_{2}^{\varphi_{0}}$ (if $\left|u^{*} v\right|=k$, then this follows from statement (2) of Theorem 5.1; otherwise this immediately follows from the definition of $\varphi_{0}$ ). On the other hand, we have $|S(v, t)|=|S(w, t)|=k$. Therefore $a_{2}^{*} \in S(v, t)$ and there exists $a_{3} \in$ $S(t, w)$ such that $c=a_{2}^{*} \cdot a_{3}$ (see Figure 7). As above one can see that $c^{\varphi_{0}}=\left(a_{2}^{*}\right)^{\varphi_{0}} \cdot a_{3}^{\varphi_{0}}$

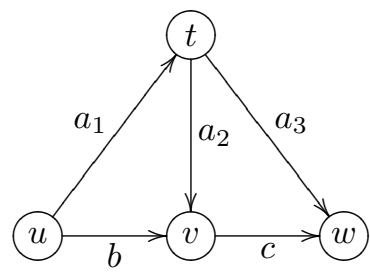

Figure 7:

and that $\left(a_{1} \cdot a_{3}\right)^{\varphi_{0}}=a_{1}^{\varphi_{0}} \cdot a_{3}^{\varphi_{0}}$. Thus

$$
\begin{gathered}
(b \cdot c)^{\varphi_{0}}=\left(a_{1} \cdot a_{2} \cdot a_{2}^{*} \cdot a_{3}\right)^{\varphi_{0}}=\left(a_{1} \cdot a_{3}\right)^{\varphi_{0}}=a_{1}^{\varphi_{0}} \cdot a_{3}^{\varphi_{0}}= \\
a_{1}^{\varphi_{0}} \cdot a_{2}^{\varphi_{0}} \cdot\left(a_{2}^{*}\right)^{\varphi_{0}} \cdot a_{3}^{\varphi_{0}}=\left(a_{1} \cdot a_{2}\right)^{\varphi_{0}} \cdot\left(a_{2}^{*} \cdot a_{3}\right)^{\varphi_{0}}=b^{\varphi_{0}} \cdot c^{\varphi_{0}}
\end{gathered}
$$

which completes the proof.

\section{Schurity and separability of equivalenced schemes}

\subsection{Schurity}

The conclusion of Theorem 5.4 gives a sufficient condition for a scheme to be schurian.

Theorem 7.1. Let $(\Omega, S)$ be a scheme such that for each $\alpha \in \Omega$ the coherent configuration $\left(\Omega, S_{\alpha}\right)$ is semiregular on $\Omega \backslash\{\alpha\}$ and its fibers are $\alpha s, s \in S$. Then $(\Omega, S)$ is a regular or Frobenius scheme.

Proof. Let $\alpha \in \Omega$. Then by Theorem 2.1 the coherent configuration $\left(\Omega, S_{\alpha}\right)$ is schurian, and hence due to (2.3) any set $\alpha s, s \in S$, is the orbit of the group $G_{\alpha}$ where $G=$ 
$\operatorname{Aut}(\Omega, S)$. Since obviously $G_{\alpha, \beta}=\operatorname{id}_{\Omega}$ for all $\alpha \neq \beta$, it suffices to verify that the group $G$ is transitive. To do this we note that semiregularity of $G_{\alpha}$ on $\Omega \backslash\{\alpha\}$ implies that

$$
k:=\left|G_{\alpha}\right|=|\alpha s|, \quad \alpha \in \Omega, s \in S^{\#} .
$$

Let $\Delta \in \operatorname{Orb}(G, \Omega)$. Since any orbit of $G$ is a disjoint union of some orbits of the group $G_{\alpha}$, the number $|\Delta|$ is divided by $k$ if and only if $\alpha \notin \Delta$. However, this is impossible if $\Delta \neq \Omega$. Thus $G$ is transitive.

Let $(\Omega, S)$ be the scheme of an affine space $\mathcal{A}$. Then from (4.3) it follows that $D(u, v)=$ $S^{\#} \backslash u v$ for all $u, v \in S^{\#}$. Therefore conditions (5.6) and (5.9) are satisfied whenever the dimension of $\mathcal{A}$ is at least 3. Thus in this case by Theorems 5.4 and 7.1 the scheme $(\Omega, S)$ is schurian. Since the scheme is imprimitive, its automorphism group is a Frobenius group in its natural permutation representation. Using properties of Frobenius groups one can prove, without using the Veblen-Young Theorem, that the scheme $(\Omega, S)$ is an affine scheme of a Desarguesian affine space.

\subsection{Separability}

Similarly to the previous subsection the conclusion of Theorem 6.2 gives a sufficient condition for a scheme to be separable.

Theorem 7.2. In the condition of Theorem 7.1 the scheme $(\Omega, S)$ is separable.

Proof. From Theorem 6.2 it follows that any algebraic isomorphism $\varphi:(\Omega, S) \rightarrow\left(\Omega, S^{\prime}\right)$ has the $\left(\alpha, \alpha^{\prime}\right)$-extension

$$
\varphi_{\alpha, \alpha^{\prime}}:\left(\Omega, S_{\alpha}\right) \rightarrow\left(\Omega^{\prime}, S_{\alpha^{\prime}}^{\prime}\right)
$$

for all $\left(\alpha, \alpha^{\prime}\right) \in \Omega \times \Omega^{\prime}$. By Theorem 5.4 the coherent configuration $\left(\Omega, S_{\alpha}\right)$ is semiregular on $\Omega \backslash\{\alpha\}$, and hence is separable (Theorem 2.1). This implies that the algebraic isomorphism $\varphi_{\alpha, \alpha^{\prime}}$ is induced by an isomorphism. Due to the right-hand side of (2.4) this shows that the same isomorphism induces the algebraic isomorphism $\varphi$. Thus any algebraic isomorphism of the scheme $(\Omega, S)$ is induced by an isomorphism and hence this scheme is separable.

\subsection{Pseudocyclic schemes}

Let $(\Omega, S)$ be an equivalenced scheme of valency $k$ and indistinguishing number $c$.

Lemma 7.3. $|\bar{D}(u, v)|<c k^{3}, u, v \in S^{\#}$ where $\bar{D}(u, v)=S^{\#} \backslash D(u, v)$.

Proof. Let $u, v \in S^{\#}$. Then it is easily seen that $\left|u u^{*} v v^{*}\right| \leq k^{3}$. Besides, given $t \in$ $u u^{*} v v^{*}, t \in S^{\#}$, there exist at most $c(t)-1 \leq c$ relations $w \in S^{\#}$ such that $t \in w w^{*}$. Thus for at most $c k^{3}$ relations $w$ the set $\left(u u^{*} v v^{*}\right) \cap w w^{*}$ contains an element $t \in S^{\#}$. This means that $|\bar{D}(u, v)|<c k^{3}$.

Suppose that the scheme $(\Omega, S)$ is pseudocyclic. Then by Theorem 3.2 we have $c=$ $k-1$. If, in addition, $|S| \geq 4 c k^{3}$, then by Lemma 7.3 conditions (5.6) and (5.9) are satisfied for all $u, v \in S^{\#}$. By Theorem 5.4 this implies that given $\alpha \in \Omega$ the $\alpha$-fission of the scheme $(\Omega, S)$ is a semiregular coherent configuration on $\Omega \backslash\{\alpha\}$ the fibers of which are $\alpha u, u \in S$. So by Theorems 7.1 and 7.2 we obtain the following statement.

Theorem 7.4. Any pseudocyclic scheme of valency $k>1$ and rank at least $4(k-1) k^{3}$ is a separable Frobenius scheme. 


\section{Miscellaneous}

\subsection{2-designs}

Any commutative pseudocyclic scheme of of valency $k$ on $n$ points produces a $2-(n, k, k-$ 1)-design [4, Corollary 2.2.8]. The same is also true in the non-commutative case (Theorem 8.1). It would be interesting to study these designs in detail.

Theorem 8.1. A scheme $(\Omega, S)$ on $n$ points is pseudocyclic of valency $k$ if and only if the pair $(\Omega, B)$ with $B=\left\{\alpha s: \alpha \in \Omega, s \in S^{\#}\right\}$ is a $2-(n, k, k-1)$-design.

Proof. The pair $(\Omega, B)$ is an $2-(n, k, k-1)$-design if and only if $|\alpha s|=k$ for all $\alpha \in \Omega$ and $s \in S^{\#}$, and the number of blocks $\alpha s \in B$ containing two distinct points $\beta, \gamma \in \Omega$ coincides with $k-1$. However, the number of these blocks is obviously equals $c(s)$ where $s=r(\beta, \gamma)$. Thus the required statement follows from Theorem 3.2.

\subsection{Point fission}

It was proved in [11, Lemma 5.13] that a one point fission of an imprimitive equivalenced scheme is close to be semiregular. The following statement shows that the imprimitivity condition can be removed for pseudocyclic schemes the rank of which is much more than the valency.

Theorem 8.2. Let $(\Omega, S)$ be a pseudocyclic scheme of valency $k$. Suppose that $|S|>$ $2 k(k-1)+2$. Then given $\alpha \in \Omega$ the coherent configuration $\left(\Omega, S_{\alpha}\right)$ is semiregular on $\Omega \backslash\{\alpha\}$.

Proof. For a relation $u \in S^{\#}$ set $E(u)=\left\{v \in S^{\#}:\left|u^{*} v\right|=k\right\}$. Since by Theorem 3.2 the indistinguishing number $c$ of the scheme $(\Omega, S)$ is $k-1$, we have

$$
|S \backslash E(u)|=\sum_{b \in u u^{*} \backslash\left\{1_{\Omega}\right\}}\left|\left\{v \in S: b \in v v^{*}\right\}\right| \leq\left|u u^{*}\right| c \leq k(k-1) .
$$

By the theorem hypothesis this implies that

$$
|E(u) \cap E(v)| \geq|S|-2(k-1) k-2>0
$$

for all non-equal $u, v \in S^{\#}$. Set $S_{0}=\left\{s \in S_{\alpha}: s \subset \Omega_{0} \times \Omega_{0}\right\}$ where $\Omega_{0}=\Omega \backslash\{\alpha\}$. To complete the proof it suffices to verify that each $s_{0} \in S_{0}$ is contained in some $s \in S_{0}^{\cup}$ for which $|\beta s| \leq 1$ for all $\beta \in \Omega$. However, it is easy to see that given $s_{0} \in S_{0}$ one can find $u, v \in S^{\#}$ such that

$$
s_{0} \subset \alpha u \times \alpha v .
$$

Due to (8.1) there exists $w \in E(u) \cap E(v)$. Since $S_{\alpha}(u, w), S_{\alpha}(w, v) \subset S_{0}^{\cup}$, we have $S_{\alpha}(u, v ; w) \subset S_{0}^{\cup}$. Thus $s_{0} \subset s$ for some $s \in S_{\alpha}(u, v ; w)$. To complete the proof it suffices to note that $|\beta s| \leq 1$ for $\beta \in \Omega$.

By the assumptions of Theorem 8.2 we have $|\beta s| \leq 1$ for all $\beta \in \Omega \backslash\{\alpha\}$ and $s \in S_{\alpha}$. Therefore by [13, Theorem 9.3] we obtain the following statement.

Corollary 8.3. In the condition of Theorem 8.2 any one point fission of the scheme $(\Omega, S)$ is schurian and separable.

We complete the subsection by making a remark that Corollary 8.3 together with [12, Theorem 4.6] shows that any pseudocyclic scheme of valency $k$ and rank $O\left(k^{2}\right)$ is 2schurian and 2-separable in the sense of [14]. 


\subsection{Affine schemes}

The following characterization of the affine schemes is known in commutative case (see [9]).

Theorem 8.4. Let $(\Omega, S)$ be a scheme with $n_{s} \geq 3$ for each $s \in S^{\#}$. Then it is the scheme of an affine space if and only if $c_{r s}^{t} \leq 1$ for all $r, s, t \in S$ such that $r \neq s^{*}$.

Proof. The necessity follows from (4.3). To prove the sufficiency let $s \in S^{\#}$. Then $\left|s s^{*}\right|>1$ because $n_{s}>1$, and $s s^{*} \cap r r^{*}=\left\{1_{\Omega}\right\}$ for all $r \neq s$ (Lemma 2.3). Thus

$$
\left|S^{\#}\right| \geq\left|\bigcup_{s \in S^{\#}} s s^{*} \backslash\left\{1_{\Omega}\right\}\right|=\sum_{s \in S^{\#}}\left|s s^{*} \backslash\left\{1_{\Omega}\right\}\right| \geq\left|S^{\#}\right| .
$$

This implies that there exists a bijection $s \mapsto s^{\prime}$ from $S^{\#}$ to itself such that $s s^{*}=\left\{1_{\Omega}, s^{\prime}\right\}$. In particular, the scheme $(\Omega, S)$ is symmetric and $c_{s^{\prime} s}^{s}=n_{s}-1 \geq 2$. Therefore $s^{\prime}=s$ for each $s \in S^{\#}$. Thus each relation from $S$ is an equivalence relation minus a diagonal. All such schemes were classified in [9] where it was proved that each of them is the scheme of an affine space.

\subsection{QI-groups}

A permutation group $G \leq \operatorname{Sym}(\Omega)$ is called $Q I[7]$ if its permutation module $\mathbb{Q} \Omega$ is a direct sum of a one dimensional module and an irreducible one, say $(\mathbb{Q} \Omega)^{0}$. Notice that $(\mathbb{Q} \Omega)^{0}$ consists of all vectors with zero sum of coordinates.

Let $K$ be the splitting field of the group algebra $\mathbb{Q}[G]$. Denote by $\mathcal{X}$ a full set of irreducible $K$-representations appearing in the decomposition of $(K \Omega)^{0}$. Then the Galois group of the extension $K / \mathbb{Q}$ acts transitively on $\mathcal{X}$. This implies that the pair $\left(n_{\chi}, m_{\chi}\right)$ does not depend on $\chi \in \mathcal{X}$ where $n_{\chi}$ and $m_{\chi}$ are respectively the degree and multiplicity of $\chi$. From this one can deduce that the scheme of the group $G$ is pseudocyclic. Moreover, by the second part of Theorem 3.2 this scheme is also commutative.

\subsection{The Terwilliger algebra of a pseudocyclic scheme}

Let $(\Omega, S)$ be an arbitrary scheme and let $T_{\alpha}(\Omega, S)$ be the Terwilliger algebra of it at point $\alpha \in \Omega$, i.e. the subalgebra of $M_{\Omega}(\mathbb{C})$ generated by $\mathbb{C} S$ and the set of matrices $A\left(1_{\alpha s}\right)$, $s \in S$. It immediately follows that

$$
T_{\alpha}(\Omega, S) \subseteq \mathbb{C} S_{\alpha}
$$

where $\mathbb{C} S_{\alpha}$ is the adjacency algebra of the scheme $\left(\Omega, S_{\alpha}\right)$ (see Subsection 2.6), and that equality holds exactly when the algebra in the left-hand side is closed with respect to the Hadamard product.

Let $G=\operatorname{Aut}(\Omega, S)$. As it was observed in [21] it is true that $T_{\alpha}(\Omega, S)$ is always contained in the centralizer algebra $\mathbb{C} G_{\alpha}$ of the one-point stabilizer $G_{\alpha}$. However, even for cyclotomic schemes this inclusion can be strict. On the other hand, due to (2.3) we have

$$
\mathbb{C} S_{\alpha} \subseteq \mathbb{C} G_{\alpha}
$$

Thus from Theorem 7.4 and the above two inclusions it follows that the Terwilliger algebra of the cyclotomic scheme of valency $k$ and rank at least $4(k-1) k^{3}$ is coherent and coincides with $\mathbb{C} G_{\alpha}$. 


\subsection{Equivalenced schemes with bounded indistinguishing number}

Theorem 7.4 may be strengthened if we replace the condition of being pseudocyclic by bounding of the indistinguishing number of a scheme. Indeed, the argument used in the proof of Theorem 7.4 yields us that a $k$-valenced scheme with indistinguishing number $c$ of rank at least $c k^{3}$ is a separable Frobenius scheme.

\section{Acknowledgments}

The authors are very grateful to P.-H. Zieschang who proposed an alternative proof of Theorem 3.2. The first author would like to thank M.Klin for fruitful conversations. The authors are thankful to an anonymous referee for valuable remarks.

\section{References}

[1] L. Babai, On the order of uniprimitive permutation groups, Annals of Math. 113 (1981), 553568.

[2] J. Bagherian and I. Ponomarenko, A. Rahnamai Barghi, On cyclotomic schemes over finite near-fields, Journal of Algebraic Combinatorics 27 (2008), 173-185.

[3] J. L. Berggren, An algebraic characterization of finite symmetric tournaments, Bull. Austral. Math. Soc. 6 (1972), 53-59.

[4] A. E. Brouwer, A. M. Cohen and A. Neumaier, Distance-regular graphs, Ergebnisse der Mathematik und ihrer Grenzgebiete, 3. Folge, 18, Springer-Verlag, Berlin, 1989.

[5] F. Buekenhout and P. Cameron, Projective and affine geometry over division rings, in: F. Buekenhout (ed.), Handbook of Incidence Geometry, Elsevier, Amsterdam, 1995, 27-62.

[6] P. J. Cameron, Permutation groups whose non-identity elements have $k$ fixed points, J. Group Theory 4 (2001), 45-51.

[7] P. Cameron, Synchronization and homomorphisms, http://www. maths . qmul.ac.uk/ $\sim$ jjc/slides/beamer/perthtalk1.pdf.

[8] C. W. Curtis and I. Reiner, Representation theory of finite groups and associative algebras, Pure and Applied Mathematics 11 (1962), New York-London: Interscience Publishers, a division of John Wiley \& Sons.

[9] E. R. van Dam, A Characterization of Association Schemes from Affine Spaces, Designs, Codes and Cryptography 21 (2000), 83-86.

[10] S. Evdokimov and I. Ponomarenko, Two inequalities for the parameters of a cellular algebra, Zapiski Nauchnykh Seminarov POMI 240 (1997), 82-95, English translation in J. Math. Sci., New York 96 (1999), 3496-3504.

[11] S. Evdokimov and I. Ponomarenko, On primitive cellular algebras, Zapiski Nauchnykh Seminarov POMI 256 (1999), 38-68, English translation in J. Math. Sci., New York 107 (2001), $4172-4191$.

[12] S. Evdokimov and I. Ponomarenko, Separability Number and Schurity Number of Coherent Configurations, Electronic J. Combin. 7 (2000), \#R31.

[13] S. Evdokimov and I. Ponomarenko, Characterization of cyclotomic schemes and normal Schur rings over a cyclic group, Algebra and Analysis 14 (2002), 11-55, English translation in St. Petersburg Math. J. 14 (2003), 189-221.

[14] S. Evdokimov and I. Ponomarenko, Permutation group approach to association schemes, European J. Combin. 30 (2009), 1456-1476. 
[15] I. A. Faradžev, M. H. Klin, M. E. Muzychuk, Cellular rings and groups of automorphisms of graphs, in: I.A. Faradžev et al. (eds): Investigations in algebraic theory of combinatorial objects, Kluwer Acad. Publ., Dordrecht, 1994, 1-152 (translation from Russian edition 1985).

[16] Ja. Ju. Gol'fand, A. V. Ivanov and M. H. Klin, Amorphic cellular rings, in: I. A. Faradžev et al. (eds.), Investigations in Algebraic Theory of Combinatorial Objects, Kluwer, Dordrecht, 1994, 167-186 (translation from Russian edition 1985).

[17] M. Hall, Jr., The theory of groups, The Macmillan Company, New York, 1959 (Russian translation, 1962).

[18] A. Hanaki and K. Uno, Algebraic structure of association schemes of prime order, J. Algebraic Combin 23 (2006), 189-195.

[19] H. D. L. Hollmann, Q. Xiang, Pseudocyclic association schemes arising from the actions of $\operatorname{PGL}\left(2,2^{m}\right)$ and $\operatorname{P\Gamma L}\left(2,2^{m}\right)$, J. Combin. Theory, A113 (2006), 1008-1018.

[20] T. Ikuta, T. Ito and A. Munemasa, On pseudo-automorphisms and fusions of association schemes, European J. Combin. 12 (1991), 317-325.

[21] H. Ishibashi, T. Ito and M. Yamada, Terwilliger Algebras of Cyclotomic Schemes and Jacobi Sums, European J. Combin. 20 (1999), 397-410.

[22] M. Klin, M. Muzychuk, C. Pech, A. Woldar and P.-H. Zieschang, Association schemes on 28 points as mergings of a half-homogeneous coherent configuration, European J. of Combin. 28 (2007), 1994-2025.

[23] B. R. McDonald, Finite rings with identity, Pure and Applied Mathematics, Vol. 28, Marcel Dekker Inc., New York, 1974.

[24] M. Muzychuk, I. Ponomarenko, On Pseudocyclic Association Schemes, arXiv:0910.0682v1 [math.CO] (2009), 1-23.

[25] D. V. Pasechnik, Skew-symmetric association schemes with two classes and strongly regular graphs of type $L_{2 n-1}(4 n-1)$, Acta Appl. Math. 29 (1992), 129-138.

[26] D. S. Passman, Solvable $\frac{3}{2}$-transitive permutation groups, J. Algebra 7 (1967), 192-207.

[27] D. Passman, Permutation groups, Benjamin, New York, 1968.

[28] B. Weisfeiler (Ed.), On the Construction and Identification of Graphs, in: Lect. Notes in Math., vol. 558, 1976.

[29] H. Wielandt, Finite permutation groups, Academic press, New York - London, 1964.

[30] P.-H. Zieschang, Theory of Association Schemes, Springer, Berlin \& Heidelberg, 2005. 